\section{INFLUÊNCIA DA SAÚDE BUCAL SOBRE A QUALIDADE DE VIDA DE ADOLESCENTES ESCOLARES}

\author{
Oral health influence on the quality of life of school adolescents \\ Influencia de la salud bucal en la calidad de vida de escolares \\ adolescentes
}

Artigo Original

\section{RESUMO}

Objetivo: Investigar o impacto da saúde bucal em relação à qualidade de vida de adolescentes escolares, associando-o às condições sociodemográficas. Métodos: Estudo de campo transversal e quantitativo desenvolvido em 2012, no município de Sumé-PB, com 184 adolescentes na faixa etária de 15 a 19 anos. Para avaliar o impacto, aplicou-se o questionário Oral Health Impact Profile (OHIP-14) aos participantes, enquanto para a obtenção dos dados referentes às condições sociodemográficas, os pais ou responsáveis responderam a um segundo questionário. Utilizou-se o teste Qui-quadrado para associar o impacto da saúde bucal sobre a qualidade de vida e as variáveis sociodemográficas pesquisadas, sendo considerados significativos com $p<0,05$. Resultados: Em geral, o impacto foi considerado fraco em 167 pesquisados (90,8\%). "Dor física" foi a dimensão na qualidade de vida mais afetada pelas questões bucais entre aquelas que resultaram em impacto médio $(22,8 \% ; n=42)$. Apenas as variáveis "Situação do imóvel" e "Acomodação" associaram-se ao impacto geral $(p<0,05)$. Os reduzidos percentuais de impacto geral forte $(1,1 \% ; n=2)$ relacionaram-se aos adolescentes cujas mães só estudaram até o ensino fundamental, ou às famílias que vivem com um salário mínimo ou menos $(1,1 \%)$. Conclusão: Observou-se que as condições de saúde bucal apresentaram um impacto negativo fraco na qualidade de vida dos adolescentes investigados. As análises das condições sociodemográficas dos indivíduos relacionadas ao impacto geral da qualidade de vida relacionada à saúde oral associaram-se as variáveis "Situação do imóvel" e "Acomodação".

Descritores: Adolescente; Qualidade de Vida; Saúde Bucal.

\section{ABSTRACT}

Objective: To investigate the impact of oral health on the quality of life of school adolescents associating it with sociodemographic conditions. Methods: Quantitative cross-sectional field study conducted in 2012 in the city of Sume, Paraiba, with 184 adolescents aged 15-19 years. The impact was assessed by applying the Oral Health Impact Profile (OHIP-14) to the participants, and data on sociodemographic conditions were obtained through a second questionnaire answered by parents or guardians. The Chi-Square test was used to associate the impact of oral health on quality of life and the study sociodemographic variables, with significant values set at $p<0.05$. Results: In general, the impact was considered weak on 167 participants (90.8\%). "Physical Pain" was the quality of life domain most affected by oral conditions among those that resulted in intermediate impact $(22.8 \% ; n=42)$. Only the variables "Property Situation" and "Accommodation" were associated with the overall impact $(p<0.05)$. The low percentages of strong overall impact $(1.1 \% ; n=2)$ were related to adolescents whose mothers had studied only up to primary education or whose families lived on one minimum wage or less. Conclusions: It was observed that oral health conditions had a weak negative impact on the quality of life of adolescents. The analysis of the sociodemographic conditions of individuals related to the overall impact of quality of life related to oral health were associated with the variables "Property Situation" and "Accommodation".
Suyene de Oliveira Paredes ${ }^{(1)}$ Oristácio de Sousa Leal Júnior ${ }^{(1)}$ Alcyone de Oliveira Paredes ${ }^{(2)}$ Jocianelle Maria Felix de Alencar Fernandes $^{(3)}$

Valdenice Aparecida Menezes ${ }^{(4)}$

1) Faculdades Integradas de Patos (FIP) Patos (PB) - Brasil

2) Universidade Federal do Maranhão (UFMA) - São Luís (MA) - Brasil

3) Universidade Federal da Paraíba (UFPB) - João Pessoa (PB) - Brasil

4) Universidade de Pernambuco (FOP/UPE) - Camaragibe (PE) - Brasil 


\section{RESUMEN}

Objetivo: Investigar el impacto de la salud bucal respecto la calidad de vida de escolares adolescentes y su asociación con las condiciones sociodemográficas. Métodos: Estudio de campo transversal y cuantitativo desarrollado en 2012 en el municipio de Sumé-PB con 184 adolescentes entre 15 y 19 años. Se aplicó el cuestionario Oral Health Impact Profile (OHIP-14) a los participantes para evaluar el impacto de la salud bucal mientras los padres o responsables contestaron un segundo cuestionario para la obtención de los datos de las condiciones sociodemograficas. Se utilizó la prueba Chi-cuadrado para la asociación del impacto de la salud bucal en la calidad de vida y las variables sociodemograficas investigadas, considerándose significativo el $p<0,05$. Resultados: En general, el impacto fue considerado débil en 167 investigados (90,8\%). "Dolor físico" fue la dimensión más afectada de la calidad de vida por cuestiones bucales entre las que resultaron en impacto medio $(22,8 \% ; n=42)$. Solamente las variables "Situación del inmueble" $y$ "Acomodación" se asociaron al impacto general $(p<0,05)$. Los reducidos porcentuales de impacto general fuerte $(1,1 \% ; n=2)$ se relacionaron con los adolescentes cuyas madres estudiaron hasta la enseñanza fundamental o con las familias que viven con un sueldo mínimo o menos $(1,1 \%)$. Conclusión: Se observó que las condiciones de salud bucal presentaron un impacto negativo débil en la calidad de vida de los adolescentes investigados. Los análisis de las condiciones sociodemográficas de los individuos relacionados al impacto general de la calidad de vida relacionada con la salud oral se asociaron con las variables "Situación del inmueble" y "Acomodación".

Descriptores: Adolescente; Calidad de vida; Salud Bucal.

\section{INTRODUÇÃO}

A infância e a adolescência são fases do ciclo de vida marcadas por grandes tensões, uma vez que representam períodos em que o indivíduo está crescendo e se desenvolvendo física e intelectualmente. A Organização Mundial de Saúde (OMS) define os limites cronológicos da adolescência entre 10 e 19 anos. Porém, na legislação brasileira, o Estatuto da Criança e do Adolescente considera criança o indivíduo com até 12 anos de idade incompletos, e adolescentes aqueles entre 12 e 18 anos de idade ${ }^{(1)}$.

Em sentido mais amplo, a adolescência é a fase de desenvolvimento caracterizada por conflitos, descobertas e intensidade na vivência. Diante desse contexto de urgências e intensidades, é comum que os jovens negligenciem medidas de autocuidado, sendo essa negligência pessoal reconhecida pelos adolescentes como causa dos seus problemas bucais ${ }^{(2)}$. Por isso, trata-se de um período considerado de risco em relação aos agravos de saúde bucal, em decorrência do precário controle do biofilme e da redução dos cuidados com a escovação dentária. Além disso, nessa fase da vida, os pais não delegam tarefas de cuidados de saúde bucal para os filhos, e estes tendem a não aceitar a interferência dos familiares nas práticas diárias de higiene ${ }^{(3)}$. Um percentual considerável de adolescentes relatou que não necessita ser lembrado por seus pais para higienizar os dentes ${ }^{(4)}$.

Para avaliar o comportamento de adolescentes, bem como as mudanças ocorridas em suas vidas e alterações socioeducativas, é importante que as avaliações sejam feitas em escolas, visto que as instituições de ensino agregam grande parte dos adolescentes e jovens da comunidade, e representam um espaço de socialização, formação e informação $0^{(5)}$.

A saúde bucal é importante em vários cenários da vida do adolescente. Fatores como aparência pessoal, sexualidade, emprego e saúde, de um modo geral, motivamno a cuidar da sua saúde bucal ${ }^{(6)}$. Para tanto, devem ser consideradas as dimensões sociais da saúde bucal e o real impacto dos agravos na qualidade de vida dos indivíduos ${ }^{(7)}$.

A análise de fatores sociodemográficos tornase relevante, visto que condições de saúde bucal são influenciadas não somente por hábitos comportamentais, mas também por questões sociais. A prevalência e incidência da cárie dentária são fortemente associadas a indivíduos pertencentes às classes sociais mais necessitadas, cujos pais possuem baixo nível de escolaridade ${ }^{(8,9)}$, assim como hábitos de higiene bucal também são influenciados pelo nível socioeconômico ${ }^{(4)}$. Condições sociais críticas nas primeiras fases da vida podem predispor indivíduos a uma condição dentária que, por sua vez, pode ter impacto negativo na qualidade de vida na adolescência. Além disso, diante do entendimento de que a saúde bucal também envolve aspectos afetivos, estéticos e sociais, faz-se necessário avaliar o impacto das condições de saúde bucal desses jovens sobre sua qualidade de vida ${ }^{(10)}$.

Em face das vulnerabilidades pelas quais essa população se expõe, a saúde do adolescente deve ser contemplada de uma maneira holística, e não voltada ao modelo biomédico que fragmenta o indivíduo, com foco apenas nas doenças. Para tanto, a Estratégia Saúde da Família (ESF), por meio das ações de saúde bucal, deve atender a essa prerrogativa ampliada de cuidados, representando um avanço para a promoção da saúde deste público. Contudo, as ações programadas ou sistematizadas ainda não atendem ao adolescente, o que configura um dos desafios para a saúde bucal no Brasil ${ }^{(11)}$.

Sob essa ótica, a escola representa um cenário imprescindível no contexto da promoção da saúde bucal, possibilitando que o autocuidado seja incentivado e repercutindo na melhora das condições de saúde bucal e de hábitos no âmbito individual, familiar e coletivo, sobretudo 
com a capacitação e o planejamento conjunto das ações entre os profissionais da escola e os profissionais de saúde ${ }^{(12)}$.

A importância da atual pesquisa, desenvolvida em um município de pequeno porte, pertencente ao Semiárido do Nordeste brasileiro, centra-se na aquisição de dados que refletem carências e necessidades da população estudada. Diante do exposto, o objetivo do presente estudo foi investigar o impacto da saúde bucal em relação à qualidade de vida de adolescentes escolares, associando-o às condições sociodemográficas.

\section{MÉTODOS}

Trata-se de estudo de campo, transversal, com abordagem quantitativa dos dados, realizado no período compreendido entre fevereiro e novembro de 2012, no município de Sumé-PB, na mesorregião da Borborema e microrregião do Cariri Ocidental. Esse município possui área territorial de $838.058 \mathrm{Km}^{2}$ e população composta por 16.060 habitantes, de acordo com os dados da última contagem populacional ${ }^{(13)}$, caracterizando-se, portanto, como um município de pequeno porte, pertencente ao Semiárido do Nordeste brasileiro.

A população constou de adolescentes na faixa etária de 15 a 19 anos, matriculados em escolas públicas de ensino fundamental e médio. Com relação à determinação do tamanho amostral, considerou-se a população finita de 571 adolescentes, erro aceitável de 5,0\%, nível de confiança de $95 \%$ e prevalência de $50,0 \%$. Para o estabelecimento dos adolescentes que constituíram a amostra, realizou-se o processo de amostragem aleatória ou probabilística do tipo sistemática. Dessa forma, a amostra inicial constou de 230 adolescentes e a amostra final totalizou 184.

Para isso, solicitou-se às autoridades de ensino uma listagem dos adolescentes regularmente matriculados, contendo as respectivas datas de nascimento. A partir do fornecimento das listas de alunos por sala, organizou-se uma listagem única por escola, incluindo apenas os indivíduos de 15 a 19 anos. Sequencialmente, as listas referentes às instituições compuseram uma relação única. Para a seleção dos adolescentes designados a participar do estudo, optouse por um intervalo de amostragem por meio de sorteio para definir o início aleatório.

Como critérios de inclusão, consideraram-se os adolescentes com idade entre 15 e 19 anos, regularmente matriculados nas instituições de ensino selecionadas, cujos pais ou responsáveis tenham assinado o Termo de Consentimento Livre e Esclarecido (TCLE) autorizando a participação voluntária do jovem na pesquisa, bem como o Termo de Assentimento do Menor, caso o adolescente possuísse idade inferior a 18 anos. Respeitando a Resolução Ética Brasileira, solicitou-se um termo de anuência das autoridades de ensino, de forma que elas permitissem a realização da pesquisa e estivessem cientes de suas corresponsabilidades para com os sujeitos da pesquisa.

Os dados foram coletados por um único pesquisador devidamente treinado, utilizando-se como instrumentos de coleta dois questionários direcionados aos adolescentes e a seus responsáveis.

Para avaliar o impacto das condições de saúde bucal sobre a qualidade de vida dos adolescentes, aplicou-se aos participantes do estudo o questionário Oral Health Impact Profile (OHIP-14), validado no idioma português ${ }^{(14)}$ e utilizado como instrumento para verificar as experiências vivenciadas pelos adolescentes nos últimos 12 meses, segundo metodologia preconizada ${ }^{(15)}$. O OHIP-14, através de 14 perguntas, permitiu avaliar os seguintes parâmetros ou dimensões na qualidade de vida: limitação funcional, dor física, desconforto psicológico, incapacidade física, incapacidade psicológica, incapacidade social e desvantagem social. As opções de resposta variaram em uma escala de códigos de 0 a 4, sendo: 0 (nunca), 1 (dificilmente), 2 (às vezes), 3 (quase sempre) e 4 (sempre). Os índices totais obtidos foram calculados a partir do somatório dos códigos das respostas, multiplicados pelos respectivos pesos das questões. Quanto menor fosse o valor obtido (mínimo $=$ zero), menor também seria o impacto negativo da saúde bucal sobre a qualidade de vida do adolescente. Contrariamente, a máxima (máximo $=28)$ representaria um maior impacto negativo das condições de saúde bucal na qualidade de vida dos indivíduos. Assim, classificou-se o impacto geral em: fraco, quando os índices obtidos variaram de 0 a 9; médio, entre 10 e 18; e forte, entre 19 e 28 . A avaliação do impacto por domínio ou dimensão seguiu a metodologia utilizada em outro estudo ${ }^{(15)}$ : fraco $(\leq 1,33)$, médio $(1,33<\mathrm{x}<2,67)$ e forte $(\geq 2,67)$.

Após o sorteio, o adolescente participante respondia ao questionário OHIP-14, formato impresso, em ambiente escolar reservado para a realização da pesquisa. Ressalta-se que após as instruções de preenchimento fornecidas pelo pesquisador, o questionário respondido era depositado pelo adolescente em uma pasta arquivo, juntamente com outros questionários, a fim de não se identificar o indivíduo e garantir o sigilo das informações, já que não continham dados de informação pessoal. Não se determinou tempo para o preenchimento do instrumento.

Para a obtenção dos dados referentes à situação sociodemográfica, tais como renda familiar, escolaridade materna ou paterna e situação de moradia, enviou-se um segundo questionário, sociodemográfico, baseado em instrumento de coleta publicado na literatura científica ${ }^{(16)}$, juntamente com o TCLE e o Termo de Assentimento do Menor, aos pais ou responsáveis, em dia anterior à aplicação dos questionários aos adolescentes. 
Os dados foram digitados e analisados no programa estatístico Statistical Program for Social Science, versão 20.0 (SPSS for Windows, SPSS, Inc., Chicago, IL, USA), com a utilização de estatística descritiva para análise dos dados e obtenção dos resultados. Para os dados quantitativos e de frequência obtidos a partir dos questionários, utilizouse o teste Qui-quadrado para associar o impacto da saúde bucal sobre a qualidade de vida com as variáveis sociodemográficas, sendo considerado significativo com $\mathrm{p}<0,05$.

O presente estudo recebeu aprovação do Comitê de Ética em Pesquisa envolvendo seres humanos das Faculdades Integradas de Patos (FIP), sob protocolo $\mathrm{n}^{\mathrm{o}}$ $167 / 2011$, em cumprimento aos requisitos exigidos pela Resolução 466/12, do Conselho Nacional de Saúde (CNS/ MS) $)^{(17)}$.

\section{RESULTADOS}

A partir do total de 230 indivíduos que compunham a amostra inicial do presente estudo, contabilizaram-se 184 adolescentes. O percentual de perda amostral representou $20 \%$, entre aqueles que se recusaram a participar e os demais que estavam ausentes no momento da coleta dos dados. Portanto, 184 participantes representaram a amostra final, com idades variando entre 15 a 19 anos, sendo a média de 15,8 anos $( \pm 1,02)$ (Tabela I).

Com relação ao sexo, observou-se maior frequência de adolescentes do sexo feminino $(57,1 \%$; $n=105)$ e $42,9 \%$ $(\mathrm{n}=79)$ de adolescentes do sexo masculino.

Os percentuais de impacto geral fraco prevaleceram sobre o impacto geral médio e forte em todas as variáveis sociodemográficas pesquisadas $-90,8 \%(\mathrm{n}=167) ; 8,2 \%$ $(n=15)$ e $1 \%(n=02)$ respectivamente. O impacto geral forte - $100 \%(\mathrm{n}=02)$ - influencia negativamente a qualidade de vida dos adolescentes e está relacionado às mães que só estudaram até o ensino fundamental, e às famílias que vivem com um salário mínimo ou menos (Tabela II).

Na Tabela II, observa-se que as variáveis "Acomodação" $(p=0,000)$ e "Situação do imóvel" $(p=0,025)$ apresentaram associação estatisticamente significante em relação ao impacto geral da saúde bucal sobre a qualidade de vida.

Os resultados relacionados às variáveis sociodemográficas mostraram considerados percentuais de pais que possuem pouca escolaridade, ou seja, a maioria das mães $(59,8 \% ; n=110)$ e dos pais $(63 \% ; n=116)$ só estudou até o ensino fundamental. Além disso, a quantidade de pais que não frequentaram a escola totalizou 19,6\% $(n=36)$ (Tabela II).

O teste estatístico do Qui-quadrado foi aplicado para a análise individual das associações entre as variáveis sociodemográficas pesquisadas e as dimensões ou domínios do OHIP-14. Assim, as variáveis "Situação do imóvel", "Localização da moradia" "Acomodação" e "Escolaridade materna" mostraram associações estatisticamente significativas, com algumas dimensões do OHIP-14: "Situação do imóvel" revelou associação estatística com "Desvantagem social" (OHIP 13 e 14) $(p=0,010)$; "Localização de moradia", com "Dor física" (OHIP 3 e 4) $(p=0,010)$; "Acomodação" relacionou-se estatisticamente com os domínios "Limitação funcional" (OHIP 1 e 2 ) $(\mathrm{p}=0,001), \quad$ "Desconforto psicológico" (OHIP 5 e 6) $(\mathrm{p}=0,001)$ e "Incapacidade física" (OHIP 7 e 8$)(\mathrm{p}=0,001)$. Quanto à escolaridade materna, a análise por dimensão mostrou significância estatística $(p=0,004)$ em "Limitação funcional" (OHIP 1 e 2) - problemas referentes à pronúncia de palavras e sensação de sabores de alimentos por causa de problemas bucais.

As demais variáveis não mostraram valores significativos, tais como "Número de pessoas residentes" $(p=0,159)$, "Total de cômodos" $(p=0,639)$, "Renda mensal familiar" $(\mathrm{p}=0,765)$ e "Escolaridade paterna" $(\mathrm{p}=0,791)$.

Em geral, o impacto negativo das condições de saúde bucal na qualidade de vida dos adolescentes pesquisados é fraco. Porém, ao se analisar o impacto da qualidade de vida relacionada à saúde bucal (QVRSB) por dimensão, observou-se que todas as dimensões ou domínios apresentaram percentuais de impacto forte, apesar de os valores obtidos terem sido consideravelmente baixos. Dentre todas as dimensões, observou-se que "Desvantagem

Tabela I - Distribuição dos adolescentes de acordo com a idade e instituição participante. Sumé-PB, 2012.

\begin{tabular}{lcccccccc}
\hline Idade (anos) & \multicolumn{2}{c}{ Instituição A } & \multicolumn{2}{c}{ Instituição B } & \multicolumn{2}{c}{ Instituição C } & \multicolumn{2}{c}{ Total } \\
\hline & $\mathbf{n}$ & $\mathbf{\%}$ & $\mathbf{n}$ & $\mathbf{\%}$ & $\mathbf{n}$ & $\mathbf{\%}$ & $\mathbf{n}$ & $\mathbf{\%}$ \\
15 & 34 & 18,5 & 13 & 7,1 & 44 & 23,9 & 91 & 49,5 \\
16 & 25 & 13,6 & 03 & 1,6 & 19 & 10,3 & 47 & 25,6 \\
17 & 15 & 8,1 & 01 & 0,5 & 15 & 8,1 & 31 & 16,8 \\
18 & 08 & 4,3 & 00 & 00 & 04 & 2,2 & 12 & 6,5 \\
19 & 01 & 0,5 & 00 & 00 & 02 & 1,1 & 03 & 1,6 \\
\hline Total & 83 & 45 & 17 & 9,2 & 84 & 45,6 & 184 & 100 \\
\hline
\end{tabular}


Tabela II - Associação entre o impacto das condições de saúde bucal na qualidade de vida e fatores sociodemográficos pesquisados. Sumé-PB, 2012.

\begin{tabular}{|c|c|c|c|c|c|c|c|c|c|}
\hline \multirow{3}{*}{$\begin{array}{l}\text { Variável } \\
\text { sociodemográfica }\end{array}$} & \multicolumn{6}{|c|}{ Impacto geral } & \multirow{2}{*}{\multicolumn{2}{|c|}{ Total }} & \multirow{3}{*}{ Valor de $p$} \\
\hline & \multicolumn{2}{|c|}{ Fraco } & \multicolumn{2}{|c|}{ Médio } & \multicolumn{2}{|c|}{ Forte } & & & \\
\hline & $\mathbf{n}$ & $\%$ & $\mathbf{n}$ & $\%$ & $\mathbf{n}$ & $\%$ & $\mathbf{n}$ & $\%$ & \\
\hline \multicolumn{10}{|l|}{ Situação do imóvel } \\
\hline Próprio & 129 & 70,1 & 13 & 7,1 & 01 & 0,5 & 143 & 77,7 & $0,025^{*}$ \\
\hline Financiado & 07 & 3,8 & 00 & 00 & 01 & 0,5 & 08 & 4,3 & \\
\hline Alugado & 22 & 12 & 00 & 00 & 00 & 00 & 22 & 12 & \\
\hline Cedido & 09 & 05 & 02 & 1,1 & 00 & 00 & 11 & 06 & \\
\hline \multicolumn{10}{|l|}{ Localização da moradia } \\
\hline Zona rural & 49 & 26,6 & 08 & 4,3 & 01 & 0,5 & 58 & 31,5 & 0,136 \\
\hline Zona urbana & 118 & 64,1 & 07 & 3.8 & 01 & 0,5 & 126 & 68,5 & \\
\hline \multicolumn{10}{|l|}{ Número de pessoas residentes } \\
\hline 01 a 03 pessoas & 30 & 16,3 & 03 & 1,6 & 01 & 0,5 & 34 & 18,4 & 0,159 \\
\hline 04 a 05 pessoas & 108 & 58,6 & 06 & 3,2 & 01 & 0,5 & 115 & 62,5 & \\
\hline Mais de 05 pessoas & 29 & 15,7 & 06 & 3,2 & 00 & 00 & 35 & 19 & \\
\hline \multicolumn{10}{|l|}{ Total de cômodos } \\
\hline 01 a 03 & 38 & 20,6 & 04 & 2,2 & 00 & 00 & 42 & 22,8 & 0,639 \\
\hline 04 a 05 & 66 & 35,9 & 08 & 4,3 & 01 & 0,5 & 75 & 40,8 & \\
\hline Mais de 05 & 63 & 34,2 & 03 & 1,6 & 01 & 0,5 & 67 & 36,4 & \\
\hline Acomodação & & & & & & & & & \\
\hline Suficiente & 146 & 79,3 & 10 & 5,4 & 00 & 00 & 156 & 84,8 & $0,000 *$ \\
\hline Insuficiente & 21 & 11,4 & 05 & 2,7 & 02 & 1,1 & 28 & 15,2 & \\
\hline \multicolumn{10}{|l|}{ Renda mensal } \\
\hline 01 salário ou menos & 116 & 63 & 13 & 7,1 & 02 & 1,1 & 131 & 71,2 & 0,765 \\
\hline 02 a 03 salários & 40 & 21,7 & 01 & 0,5 & 00 & 00 & 41 & 22,3 & \\
\hline 04 ou mais salários & 01 & 0,5 & 00 & 00 & 00 & 00 & 01 & 0,5 & \\
\hline Outra & 10 & 5,4 & 01 & 0,5 & 00 & 00 & 11 & 06 & \\
\hline \multicolumn{10}{|l|}{ Escolaridade materna } \\
\hline Não estudou & 13 & 7,1 & 01 & 0,5 & 00 & 00 & 14 & 7,6 & 0,977 \\
\hline Ensino fundamental & 98 & 53,3 & 10 & 5,4 & 02 & 1,1 & 110 & 59,8 & \\
\hline Ensino médio & 44 & 23,9 & 03 & 1,6 & 00 & 00 & 47 & 25,5 & \\
\hline Ensino superior incompleto & 07 & 3,8 & 01 & 0,5 & 00 & 00 & 08 & 4,3 & \\
\hline Graduado & 05 & 2,7 & 00 & 00 & 00 & 00 & 05 & 2,7 & \\
\hline \multicolumn{10}{|l|}{ Escolaridade paterna } \\
\hline Não estudou & 31 & 16,8 & 04 & 2,2 & 01 & 0,5 & 36 & 19,6 & 0,791 \\
\hline Ensino fundamental & 107 & 58,1 & 08 & 4,3 & 01 & 0,5 & 116 & 63 & \\
\hline Ensino médio & 26 & 14,1 & 03 & 1,6 & 00 & 00 & 29 & 15,8 & \\
\hline Ensino superior incompleto & 01 & 0,5 & 00 & 00 & 00 & 00 & 01 & 0,5 & \\
\hline Graduado & 02 & 1,1 & 00 & 00 & 00 & 00 & 02 & 00 & \\
\hline
\end{tabular}

*Qui-quadrado

Tabela III - Distribuição dos participantes segundo impacto das condições de saúde bucal na qualidade de vida (OHIP-14), por dimensão, dos escolares de 15 a 19 anos, das três localidades pesquisadas. Sumé-PB, 2012.

\begin{tabular}{lcccccccc}
\hline Dimensão do OHIP-14 & \multicolumn{2}{c}{ Fraco } & \multicolumn{2}{c}{ Médio } & \multicolumn{3}{c}{ Forte } & \multicolumn{2}{c}{ Total } \\
\cline { 2 - 9 } & $\mathrm{n}$ & $\%$ & $\mathrm{n}$ & $\%$ & $\mathrm{n}$ & $\%$ & $\mathrm{n}$ & $\%$ \\
\hline Limitação funcional & 167 & 90,8 & 13 & 7,1 & 04 & 2,2 & 184 & 100 \\
Dor física & 137 & 74,5 & 42 & 22,8 & 05 & 2,7 & 184 & 100 \\
Desconforto psicológico & 158 & 85,9 & 20 & 10,9 & 06 & 3,3 & 184 & 100 \\
Incapacidade física & 156 & 84,8 & 23 & 12,5 & 05 & 2,7 & 184 & 100 \\
Incapacidade Psicológica & 157 & 85,3 & 21 & 11,4 & 06 & 3,3 & 184 & 100 \\
Incapacidade social & 174 & 94,6 & 07 & 3,8 & 03 & 1,6 & 184 & 100 \\
Desvantagem social & 163 & 88,6 & 15 & 8,1 & 06 & 3,3 & 184 & 100 \\
Impacto geral & 167 & 90,8 & 15 & 8,1 & 02 & 1,1 & 184 & 100 \\
\hline
\end{tabular}


social", "Desconforto psicológico" e "Incapacidade psicológica" obtiveram o maior porcentual de impacto forte (3,3\%), enquanto "Dor física" foi o domínio que apresentou maior impacto médio (22,8\%), conforme demonstra a Tabela III.

\section{DISCUSSÃO}

A qualidade de vida pode ser definida como a percepção do indivíduo sobre sua posição na vida, no contex to da cultura e nos sistemas de valores nos quais ele vive, e em relação a seus objetivos, expectativas, padrões e preocupações ${ }^{(18)}$. A qualidade de vida alta, no que diz respeito aos aspectos físicos e mentais, foi comprovada entre adolescentes que possuíam melhores condições de renda, não consumiam bebidas alcoólicas e tabaco, praticavam exercícios físicos frequentemente e não exerciam atividades trabalhistas nessa fase de vida ${ }^{(19)}$.

$\mathrm{Na}$ área odontológica, as repercussões de alterações na cavidade bucal podem interferir no bem-estar físico e psicossocial dos indivíduos ${ }^{(20,21)}$. Aspectos bucais como má oclusão, traumatismo, cárie e erosão dentária provocam repercussões na qualidade de vida dos adolescentes ${ }^{(22)}$. Além disso, as doenças periodontais têm os piores impactos prevalentes nos pacientes mais severamente acometidos ${ }^{(23)}$. Estudos comprovam que adolescentes com maiores índices de dentes cariados ou problemas periodontais apresentam maior impacto negativo no desenvolvimento de suas atividades diárias. Por outro lado, esses estudos demonstraram correlação inversa entre as maiores médias de dentes hígidos com o impacto ${ }^{(13,24)}$.

No presente estudo, observou-se uma média de idade dos indivíduos de 15,8 anos $( \pm 1,02)$. Outros estudos sobre QVRSB de adolescentes revelaram médias semelhantes: $16,1( \pm 0,90)^{(20)} ; 13,58$ anos $( \pm 1,4)^{(25)} ;(15,2$ anos e 14,9 anos) ${ }^{(26)}$. A obtenção de informações relacionadas à idade dos adolescentes é importante, visto que diferenças estatisticamente significativas foram encontradas analisando-se o padrão de higiene bucal e o impacto na qualidade de vida de adolescentes de 16 a 21 anos, comparando esse grupo aos adolescentes mais novos (12 a 15 anos) $)^{(26)}$.

Com relação ao sexo, o presente estudo identificou maior percentual de indivíduos do sexo feminino $(57,1 \%)$, diferentemente de estudo com $55,42 \%$ de adolescentes do sexo masculino ${ }^{(25)}$.

Os dados do presente estudo referentes à associação entre as variáveis sociodemográficas e o impacto geral revelaram que "Situação do imóvel" e "Acomodação" mostraram significância estatística. Notou-se que, para a maioria dos adolescentes, o tipo de acomodação foi considerado suficiente ( $\mathrm{n}=156 ; 84,8 \%$ ), associado ao maior percentual $(79,3 \%)$ de impacto fraco para essa variável. Apesar da variável "Renda mensal familiar" não ter sido associada ao impacto na atual pesquisa, comprova-se, em outro estudo ${ }^{(27)}$, a associação entre uma maior prevalência de dor de dente - agravo que acarreta impactos como diminuição das horas de sono, não realização de atividades diárias, restrições alimentares e diminuição do rendimento de trabalho - em adolescentes pertencentes às famílias com rendimento de até dois salários mínimos ${ }^{(27)}$.

Um dado preocupante revelado no presente estudo mostrou os percentuais de pais e mães com baixa escolaridade: $63 \%$ e $59,8 \%$, respectivamente. A escolaridade paterna constatou, ainda, que $19,6 \%$ dos pais eram analfabetos. Quanto à escolaridade materna, a análise por dimensão mostrou associação estatisticamente significativa com "Limitação funcional" (problemas referentes à pronúncia de palavras e sensação de sabores de alimentos por causa de problemas bucais). Os maiores valores médio e forte para esse domínio foram associados ao fato de as mães possuírem apenas o ensino fundamental, corroborando com um estudo que também associou o impacto com o baixo nível educacional de pais de adolescentes escolares $^{(26)}$. Diferentemente do presente estudo, o nível de escolaridade e ocupação dos familiares de adolescentes gregos não mostrou correlação com o OHIP-14 ou suas dimensões ${ }^{(20)}$.

Apesar de o impacto geral fraco ter prevalecido no atual estudo, demonstrando que a saúde bucal dos indivíduos pesquisados pouco influencia de forma negativa na qualidade de vida deles, há adolescentes que necessitam de uma maior atenção, justificada nos relatos que traduziram impacto geral forte. Diante do percentual de jovens que apresentaram impacto geral fraco (90,8\%), possivelmente, na localidade pesquisada, há o desenvolvimento de ações preventivas e educativas em saúde bucal, como também tratamento odontológico, abrangendo, de forma efetiva, a maioria dos adolescentes escolares.

Em pesquisa semelhante, também se encontrou considerável impacto geral fraco $(87,57 \%)^{(13)}$. Porém, outro estudo, que utilizou instrumento de medida diferente, constatou, assim como na presente pesquisa, maiores percentuais favoráveis, ou seja, a qualidade de vida relacionada à saúde oral (QDVRSO) foi boa para 60,06\% dos adolescentes; fraca para $22,6 \%$ e razoável para $17,34 \%$. Percebe-se que na escala QDVRSO a condição fraca é intermediária ${ }^{(25)}$.

Comparando-se os aspectos na qualidade de vida mais afetados pelas questões bucais, o presente estudo constatou as seguintes dimensões: "Dor física" (OHIP3 e 4) - relacionada ao questionamento de ter sentido dor ou desconforto por causa de problemas bucais, com $22,8 \%$ de 
impacto médio; "Desconforto psicológico" (OHIP 05 e 06), "Incapacidade psicológica" (OHIP 09 e 10) e "Desvantagem social" (OHIP13 e 14) - relacionadas a menos chances na vida e incapacidade de agir por causa de problemas bucais, com 3,3\% de impacto forte, corroborando com resultados em que "Dor física" e "Incapacidade psicológica" foram as manifestações negativas mais frequentes de impacto sobre a qualidade de $\operatorname{vida}^{(13)}$.

$\mathrm{Na}$ pesquisa em questão, os achados que evidenciam a baixa escolaridade dos pais corroboram com outro estudo $^{(28)}$ no qual os pais foram os mais citados $(78,40 \%)$ pelos adolescentes como os responsáveis pela transmissão de conhecimentos e práticas de higiene, como ensinar a escovar os dentes. Fica evidente, portanto, que a saúde bucal de um adolescente pode ser um bom indicador de sua saúde geral e sofre influência direta da educação familiar ${ }^{(28)}$.

A motivação e a educação em saúde bucal são ferramentas poderosas que, quando empregadas de maneira agradável e atrativa na transmissão de informações, apresentam-se eficazes na promoção de saúde. Além disso, a inclusão dos adolescentes em programas preventivos direcionados a eles dentro da ESF, por meio das ações da saúde bucal, junto com uma abordagem cuidadosa, criteriosa e sensível por parte do profissional cirurgiãodentista, pode contribuir para que cheguem à vida adulta de maneira saudável ${ }^{(29)}$.

Uma limitação do presente estudo centra-se no fato de que o exame clínico para avaliação das condições bucais não constituiu uma proposta deste. Dessa forma, a amostra pode ter sido constituída por adolescentes com ou sem experiência de cárie dentária. Nesse contexto, resultados mais preocupantes poderiam ter sido identificados, caso o impacto da saúde bucal na qualidade de vida fosse associado aos jovens com atividade passada ou atual da doença cárie. Diante do exposto, há necessidade de mais estudos direcionados aos jovens brasileiros, conduzidos nas diferentes regiões, com amostras maiores, que possam ser representativos para a população de adolescentes no país.

\section{CONCLUSÃO}

Observou-se que as condições de saúde bucal apresentaram um impacto negativo fraco na qualidade de vida dos adolescentes investigados.

"Desconforto psicológico", "Incapacidade psicológica" e "Desvantagem social" apresentaram-se como os aspectos na qualidade de vida mais afetados pelas questões bucais, observados na minoria de jovens que traduziram algum impacto forte para essas dimensões.

As análises das condições sociodemográficas dos indivíduos relacionadas ao impacto geral da qualidade de vida relacionada à saúde oral associaram-se às variáveis "Situação do imóvel" e "Acomodação".

\section{REFERÊNCIAS}

1. Ministério da Saúde (BR). Atenção psicossocial a crianças e adolescentes no SUS: tecendo redes para garantir direitos. Brasília : Ministério da Saúde; 2014.

2. Barbosa TS, Castelo PM, Leme MS, Gavião MBD. Associations between oral health-related quality of life and emotional status in children and preadolescents. Oral Dis. 2012; 18(7):639-47.

3. Brennan DS, Spencer AJ. Dental visiting history between ages 13 and 30 years and oral healthrelated impact. Community Dent Oral Epidemiol. 2014;42(3):254-62.

4. Freire MD, Jordão LM, Malta DC, Andrade SS, Peres MA. Socioeconomic inequalities and changes in oral health behaviors among Brazilian adolescents from 2009 to 2012. Rev Saúde Pública. 2015;49:1-10.

5. Hedge S, Panwar S, Bolar DR, Sanghavi MB. Characteristics of occlusion in primary dentition of preschool children of Udaipur, India. Eur J Dent. 2012;6(1):51-5.

6. Silveira MF, Martins AMEBL, Santos Neto PE, Oliveira PEA, Almeida JC, Freire RS, et al. Adolescentes: uso de serviços odontológicos, hábitos e comportamentos relacionados à saúde e autopercepção das condições de saúde bucal. Unimontes Científica. 2012;14(1):17085.

7. Paula JS, Meneghim MC, Pereira AC, Mialhe FL. Oral health, socio-economic and home environmental factors associated with general and oral-health related quality of life and convergent validity of two instruments. BMC Oral Health. 2015;15:26.

8. Peres KG, Cascaes AM, Leão AT, Côrtes MI, Vettore MV. Aspectos sociodemográficos e clínicos da qualidade de vida relacionada à saúde bucal em adolescentes. Rev Saúde Pública. 2013;47(Supl 3): 1928.

9. Vieira APGF, Jamile PK. Impacto da atenção odontológica na saúde bucal de crianças. Rev Bras Promoç Saúde. 2012;24(1):10-5.

10. Casemiro JP, Fonseca ABC, Secco FVM. Promover saúde na escola: reflexões a partir de uma revisão sobre saúde escolar na América Latina. Ciênc Saúde Coletiva. 2014;19(3):829-40.

11. Kusma SZ, Moysés ST, Moysés SJ. Promoção da saúde: perspectivas avaliativas para a saúde bucal na atenção 
primária em saúde Health promotion: perspectives for evaluation of oral health in primary healthcare. Cad Saúde Pública. 2012;28(Supl):9-19.

12. Leite CT, Vieira RP, Machado CA, Quirino GDS, Machado MFAS. Prática de educação em saúde percebida por escolares. Cogitare Enferm. 2014;19(1):13-9.

13. Ministério do Planejamento (BR). Orçamento e Gestão: Sinopse do Censo Demográfico 2010. Rio de Janeiro: IBGE; 2011.

14. Barbosa TS, Gavião MB. Quality of life and oral health in children - Part II: Brazilian version of the Child Perceptions Questionnaire. Ciênc Saúde Coletiva. 2011;16(7):3267-76.

15. Bastos R, Carvalho ES, Xavier A, Caldana ML, Bastos JRM, Lauris JRP. Dental caries related to quality of life in two Brazilian adolescent groups: a cross-sectional randomised study. Int Dent J. 2012;62(3):137-43.

16. Abanto J, Carvalho TS, Mendes FM, Wanderley MT, Bönecker M, Raggio DP. Impact of oral diseases and disorders on oral health-related quality of life of preschool children. Community Dent Oral Epidemiol. 2011;39(2):105-14.

17. Conselho Nacional de Saúde (BR). Resolução No 466, de 12 de dezembro de 2012. Diário Oficial da União; Brasília; 2013 Jun 13; Seção 1, p. 59.

18. Floriano F, Mda CC, Sheiham A, Tsakos G, Vianna MI, Cabral MB, et al. Relationship between clinical dental status and the perception of oral health-related quality of life in adults, Brazil. J Epidemiol Community Health. 2011;6 (31):A431.

19. Silveira MF, Almeida JC, Freire RS, Ferreira RC, Martins AEBL, Marcopito LF. Qualidade de vida entre adolescentes: estudo seccional empregando o SF-12. Ciênc Saúde Coletiva. 2013;18(7):2007-15.

20. Papaioannous W, Oulis CJ, Latsu D, Yfantopoulos J. Oral health related quality of life of Greek adolescents: a cross-sectional study. Eur Arch Paediatr Dent. 2011;12(3):146-50.

21. Naito M, Yuasa H, Nomura Y, Nakayama T, Hamajima N, Hanada N. Oral health status and health-related quality of life: a systematic review. J Oral Sci. 2006;48(1):1-7.
22. Oliveira DC, Pereira PN, Ferreira FM, Paiva SM, Fraiz FC.Impacto Relatado das Alterações Bucais na Qualidade de Vida de Adolescentes: revisão Sistemática. Pesq Bras Odontopediatria Clin Integr. 2013;13(1):123-9.

23. Lopes MWF, Gusmão ES, Alves RV, Cimões R. Impacto das doenças periodontais na qualidade de vida. RGO. 2011;59 (Supl):39-44

24. Krisdapong S, Prasertsom P, Rattanarangsima K, Adulyanon S, Sheiham A. Setting oral health goals that include oral health-related quality of life measures: a study carried out among adolescents in Thailand. Cad Saúde Pública. 2012;18(10):1881-92.

25. Bica I, Cunha M, Costa JS, Rodrigues V, Neves D, Albuquerque I, et al. Percepção de Satisfação Corporal em Adolescentes e a relação com a sua Saúde Oral. Millenium. 2011;40:115-31.

26. Mbwalla HS, Masalu JR, Astrøm AN. Sociodemographic and behavioural correlates of oral hygiene status anda oral health related quality of life, the Limpopo-Arusha school health Project (LASH): a cross-sectional study. BMC Pediatriccs. 2010;10:87.

27. Oliveira BA, Biazevic MGH, Michel-Crosato E. Prevalência de dor de dente, cárie dental e condições sócio-econômicas: um estudo em adultos jovens brasileiros. Odonto. 2011;19(38):7-14

28. Silva RT, Freixinho ABS, Miasato JM. Verificação do conhecimento e hábitos de saúde bucal em adolescentes de uma escola particular. Rev Odontol Univ Cid São Paulo. 2012;24(1):19-25.

29. Figueiredo PBA, Souza MV, Ota TMMN, Ribeiro BBS. Efetividade de website de educação em saúde bucal para adolescentes. Rev Bras Promoç Saúde. 2014;27(3):398-405.

\section{Endereço para correspondência:}

Suyene de Oliveira Paredes

Faculdades Integradas de Patos (FIP)

Rua: Horácio Nóbrega, S/N

Bairro: Belo Horizonte

CEP: 58704-000 - Patos - Paraíba - Brasil.

E-mail: suyparedes@hotmail.com 